\title{
Optical Transport Network Design for 5G Fixed Wireless Access
}

\author{
Chathurika Ranaweera ${ }^{1}$, Paolo Monti ${ }^{2}$, Björn Skubic ${ }^{3}$, Elaine Wong ${ }^{1}$, Marija Furdek $^{2}$, \\ Lena Wosinska ${ }^{2}$, Carmen Mas Machuca ${ }^{4}$, Ampalavanapillai Nirmalathas ${ }^{1}$, and Christina Lim ${ }^{1}$ \\ ${ }^{1}$ The University of Melbourne, Australia. ${ }^{2}$ Chalmers University of Technology, Sweden. \\ ${ }^{3}$ Ericsson Research, Sweden. ${ }^{4}$ Technical University of Munich, Germany.
}

\begin{abstract}
The fifth generation of mobile technology, 5G is anticipated to be a significant leap in the evolution of mobile communication. 5G will be designed to attain 1000 times higher data volumes, 10 times lower latency, and 100 times more connected devices than its predecessor, 4G. Due to 5 Gs ability to sustain high bandwidth per unit area, 5G is considered to be a cost-efficient solution to provide Fixed Wireless Access (FWA) to households on a large scale. FWA is seen as an attractive alternative for fixed broadband access in scenarios where last mile access based on wired technologies is not economically viable. Whilst approaches for enhancing user experience in a $5 \mathrm{G}$ FWA environment are investigated in the research community, the problem of providing cost-effective high capacity transport for FWA deployments still remains a major challenge. This is particularly challenging due to diverse transport network architectures and requirements imposed by different $5 \mathrm{G}$ deployment models. This paper addresses this problem by formulating a generalized joint-optimization framework to simultaneously plan wireless access and optical transport for 5G FWA networks in order to minimize the deployment cost whilst meeting various network requirements. We demonstrate the applicability of the proposed framework by applying it to a real scenario with a range of deployment options and where different types of optical x-haul solutions are considered. The results provide a cornerstone for deployment strategies that will be imperative for realizing a future-proof and cost-effective broadband access network.
\end{abstract}

Index Terms-Optical networks; 5G wireless network; FWA; network optimization.

\section{INTRODUCTION}

$\mathbf{F}$ ixed wireless access (FWA) is a technique used to provide fixed broadband services to end users via wireless technologies. FWA is a cost-effective approach in scenarios where the deployment of wired solutions, e.g., fiber to the home (FTTH), might not be economically viable [1]. In addition, FWA also provides other advantages including shorter deployment cycles. Given the high capacity and low latency features required by the next-generation wireless technologies such as 5G [2], FWA is gaining traction as a prominent concept to enable a fully connected society [1]. As a result, FWA systems are one of the options considered by government bodies and Telco carriers around the globe (including Australia, Europe, and USA) that are planning broadband roll-outs to solve the issues of digitaldivide [3].

Given the mass volume of wireless cells that will be deployed in a 5G network, transporting huge amounts of data between thousands of cells and the network core with low-latency in a cost-effective manner is a major challenge [4]. In this scenario, the architecture of a backhaul/fronthaul (x-haul) network and its capacity requirements depend on the deployment model of the radio access network (RAN). With the introduction of RAN architectures such as centralized/cloud RAN (C-RAN) [5] and Fog-RAN (F-RAN) [6], the choice of which Baseband Unit (BBU) functions should be deployed centrally, i.e., at the central office (CO), and which functions should be placed at the cell sites, i.e., at the Remote Radio Heads (RRHs), provides an additional parameter for the cost and network performance optimization.

More precisely, the choice of a specific functional split determines the capacity and latency requirements of the $x$-haul [7]. As a result, different types of optical technologies and architectures, e.g., point to point (PtP) optical links and point to multipoint (PtMP) passive optical networks (PON), can be used in the $x$-haul [8]. In addition, FWA is serving fixed users. As a result, functionalities such as handover are not needed, and the use of cooperative communication functions would be minimal. Therefore, the requirement of centralizing all baseband processing functionalities is relaxed under FWA deployments in comparison to when $5 \mathrm{G}$ is used to serve mobile users. In light of the above, determining which $\mathrm{x}$-haul architecture should be used in a FWA network to provide cost-effective and quality of service (QoS) guaranteed access is a crucial aspect to be addressed.

5G FWA networks consist of a high number of densely deployed cells with fairly small coverage areas in order to provide sufficiently high data rates. As a result, a large number of optical $x$-haul links will have to be available for the $x$-hauling of these cells. Therefore, there is a need for a cost-effective deployment strategy that is able to satisfy the requirements of both the wireless and the optical transport segments of a FWA network. In the literature there are a number 


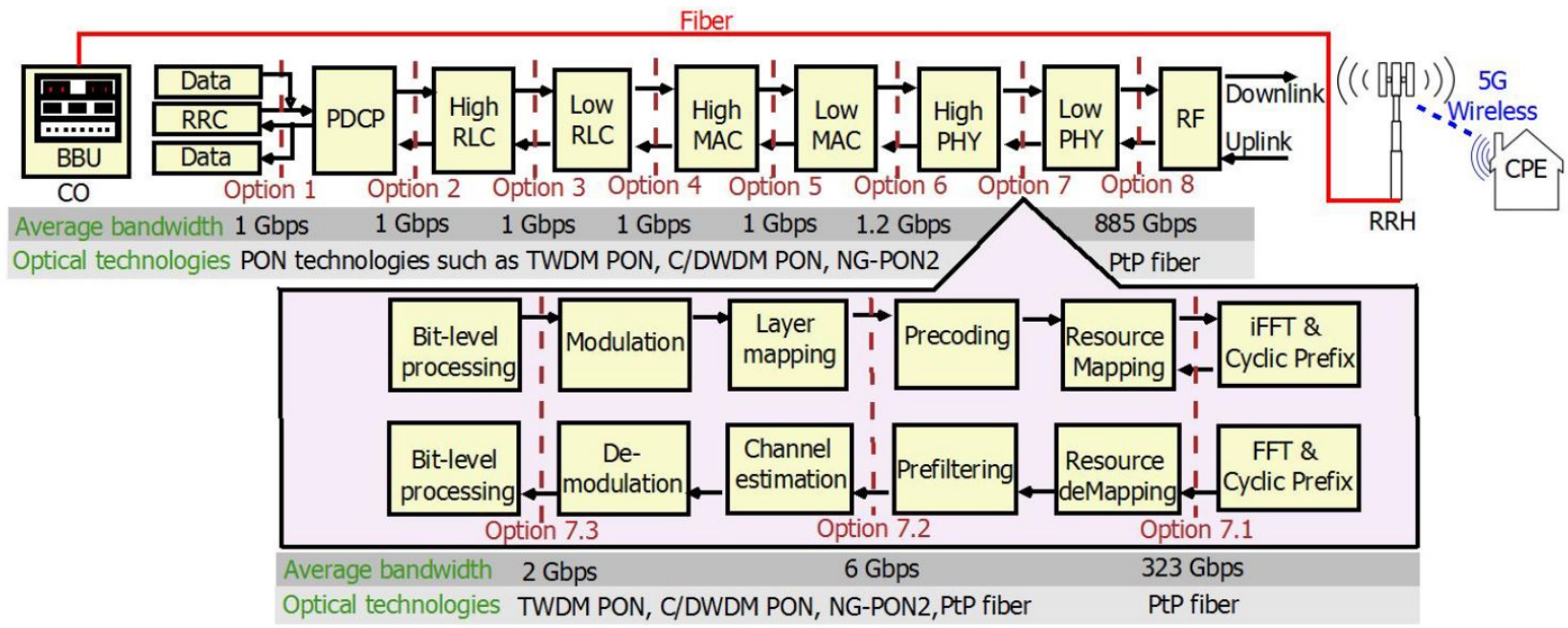

Fig. 1. Schematic view of a FWA network and possible functional splits.

of studies that investigate how to enhance the user experience in optical wireless integrated networks in the context of 5G [9]-[11]. However, the cost-optimal deployment of the wireless and transport segments of a FWA network received minimal attention so far.

This paper provides a comprehensive analysis of how the deployment of the wireless and optical x-haul segment of a 5G FWA network can be jointly optimized with respect to cost while considering: $(i)$ different RAN functional splits, and (ii) diverse requirements in terms of wireless coverage and capacity. As a result, we develop a generalized framework that can be used to jointly plan and optimize the deployment of a 5G FWA network. The proposed optimization framework is then used to solve a practical use case, i.e., the planning of a FWA network in a suburban area of Eastern Australia considering a range of deployment scenarios.

The rest of the paper is organized as follows. A discussion on the different $\mathrm{x}$-haul architectures and optical technologies that can be considered for FWA network deployment and research in progress is presented in Section II. Section III shows the details of the proposed optimization framework. In Section IV, we use the optimization framework to analyze the optimal solutions for different deployment scenarios as well as different optical $x$-haul options. The paper is concluded in Section V with a summary of our findings.

\section{5G FWA Network ARChitectURES AND VARIOUS X-HAUL OPTIONS}

The FWA configuration considered in this study and the different functional splits that can be implemented in a FWA network are shown in Fig. 1. In a FWA network, the customer premises equipment (CPE) is mounted either on the roof or on the side of a house/building and it is connected to a $\mathrm{RRH} /$ cell via a 5G wireless link. The RRH is then connected to the $\mathrm{BBU}$ placed at the $\mathrm{CO}$ via an optical $\mathrm{x}$-haul network.
The capacity required in the optical $\mathrm{x}$-haul depends on the functional split between the BBU and RRH [8], [12]. Figure 1 shows different functional split options. The $\mathrm{x}$-haul bandwidth requirement of each functional split is determined by few factors. For example, the bandwidth requirements in Options 7-2 and 7-3 ascend with both number of streams and the wireless bandwidth in use and hence rely on the number of active users and their traffic. In contrast, the capacity requirements in options 7-1 and 8 are independent of user traffic and ascend with the number of antenna ports and the wireless bandwidth. The capacity requirements of each split option shown in Fig. 1 are calculated based on 5G system that operates with 200 $\mathrm{MHz}$ of bandwidth, 64 QAM, MIMO antenna array of $8 \mathrm{X} 12$, and 96 antenna ports [8].

For example (as shown in Fig. 1), if Option 1 is selected a 1 Gbps capacity is required in the $x$-haul because most of the baseband processing functions are placed at the cell site. However, if Option 8 is selected, in a $5 \mathrm{G}$ scenario, more than $800 \mathrm{Gbps}$ are required in the $\mathrm{x}$-haul link [8] because the baseband processing takes place at the centralized BBU. As illustrated in Fig. 1, with split options up to 6 , a transport solution that supports data rates in the range of $1 \mathrm{Gbps}$ per cell is sufficient for the FWA deployment. Therefore, PtMP PON technologies, including variations of wavelength division multiplexing (WDM) PONs (e.g., coarse WDM (CWDM) PON, dense WDM (DWDM) PON, and Time WDM (TWDM) PON (NGPON2)) can be used without any critical impact on latency. This is because each wireless cell can be assigned a unique wavelength for its communication. On the other hand, for Options 7 and 8, high capacity PtP optical links are required for the $\mathrm{x}$-haul segment.

Therefore, with the development of 5G, the design of a cost-optimized FWA network is increasingly placed under scrutiny by both academia and industry [8], 
[13]. The majority of the investigations carried out focus on enhancing the wireless connectivity of FWA networks [11], [13]. For example, authors in [13] investigated an approach to enhance the link budget in a non-line-of-sight (NLOS) path between a RRH and the CPE of 5G FWA system.

In addition to the investigations addressing the wireless segment, there are few research works published on the transport part of a 5G FWA network. In [8], the authors studied the requirements of the optical transport networks of the FWA deployment. They analyzed (via simulations) some important characteristics of the FWA environment such as the required cell density, i.e., to provide the expected data rate, and the optical technology options for the FWA transport network implementation. In a scenario where a limited number of wireless access points have fiber backhaul, the authors in [14] investigated the advantages of using an access-integrated wireless backhaul in a FWA network, i.e., when the backhaul network shares the radio resources with the FWA. Their analysis suggests that in such a scenario data rates of up to $40 \mathrm{Mbps}$ can be achieved when access-integrated wireless backhaul is used in conjunction with fiber backhaul.

The planning of 5G FWA networks received minimal attention so far. In [15], a mathematical model to automate the selection and configuration of the wireless base stations of a FWA network was investigated. When developing their mathematical model the authors only considered the constraints imposed by the wireless network. Moreover, the work presented in [16] formulated a mathematical framework for planning both the coverage and the capacity of a FWA network but considering only the radio constraints.

Furthermore, a few studies on the optimal design and dimensioning of PON in greenfield scenario without considering wireless access network deployments are also reported in the literature [17]-[20]. For example, authors in [18] investigated an optimization scheme for the deployment of greenfield time division multiplexing (TDM)/WDM PONs to minimize the overall deployment cost. The framework proceeds in three phases to provide cost-optimal PON deployment whilst accommodating network traffic demand.

As seen in the literature, there is a significant gap in terms of a proper planning framework for 5G FWA networks. This is because, during the planning phase, the constraints from the transport network should be taken into account in addition to the wireless ones as the transport network can cause major implications on the cost and performance. Having this idea in mind, we investigated how we can optimally choose the most cost-effective optical transport technology for the deployment of a FWA network [21]. In this paper, we present an Integer Linear Programming (ILP)-based joint optimization framework that can be applied in any given scenario to simultaneously plan a costoptimal 5G FWA and its optical x-haul network. As
TABLE I

DATA SET

\begin{tabular}{ll}
\hline \hline Notation & Description \\
\hline $\mathbf{C}$ & A set of locations (existing COs) where the Optical \\
& Line Terminals (OLTs) and BBUs are placed \\
M & A set of locations (fiber access points, FAPs) where \\
& we have access to existing fiber and hence, can place \\
& splitters/MUXs in the network \\
A set of locations where RRHs and ONUs can be placed, \\
B & A shich we call a node \\
& provide 5G FWA service \\
\hline \hline
\end{tabular}

inputs of our framework, we consider the deployment costs of both the 5G FWA and its x-haul network, the geographic/network locations of equipment and household, and a range of network requirements including transport network capacity, user capacity, and user coverage. The framework provides the cost-optimal solution by identifying the optimal locations of the RRH/Optical Network Units (ONUs) that satisfy the coverage and capacity requirements, the optimal fiber routes that leverage the existing fiber resources to deploy the $\mathrm{x}$-haul network, and the optimal locations to deploy optical splitters, BBUs, and CO equipment.

\section{iII. Optimal FWA Network Planning: Problem FORMULATION}

We formulate an ILP-based optimization framework with the objective of minimizing the total deployment

TABLE II

NETWORK PARAMETERS

\begin{tabular}{ll}
\hline \hline Notation & Description \\
\hline$n_{C}$ & Number of existing COs available for the BBU and \\
& OLT placement, $n_{C}=|\mathbf{C}|$ \\
$n_{M}$ & Number of FAPs, $n_{M}=|\mathbf{M}|$ \\
$n_{O}$ & Number of nodes available for the RRHs and ONUs \\
& deployment $n_{O}=|\mathbf{O}|$ \\
$n_{B}$ & Number of households, $n_{B}=|\mathbf{B}|$ \\
$d f_{i, j}$ & Distance between $i^{t h}$ CO and $j^{\text {th }}$ FAP, \\
& where $i \epsilon \mathbf{C}, j \in \mathbf{M}$ \\
$d d_{j, k}$ & Distance between $j^{\text {th }}$ FAP and $k^{t h}$ node, where \\
& $j \epsilon \mathbf{M}, k \epsilon \mathbf{O}$ \\
$t_{k, l}$ & $t_{k, l}=1$, if the $l^{t h}$ household is within a given \\
& radius from the $k^{t h}$ node; \\
& $t_{k, l}=0$, otherwise; where $k \epsilon \mathbf{O}, l \in \mathbf{B}$. \\
$d_{m a x}$ & Maximum transmission distance of a PON \\
$n_{s}$ & Number of splits per splitters (split ratio) \\
$n_{p}$ & Number of PONs/OLTs supported by one line card \\
$n_{r}$ & Maximum number of RRHs deployed in one location \\
$p_{c}$ & Required percentage of household coverage, \\
& where $0 \leq p_{c} \leq 100$ \\
$c_{h}$ & Capacity requirement of a household \\
$c_{r}$ & Capacity provided by an RRH \\
$b i g M$ & A large integer \\
\hline \hline
\end{tabular}


cost of 5G FWA and its optical x-haul networks whilst satisfying other important network constraints including household coverage, capacity, and connectivity. We model the framework to satisfy the requirements of PtMP optical x-haul deployment. However, the framework can be easily adapted to plan PtP optical $\mathrm{x}$-haul deployments by omitting a few cost values and constraints. The formulation of the optimization framework is as follows.

\section{A. Data sets and network parameters}

In our framework we use four different datasets to represent various network locations. These sets and their descriptions are listed in Table I. The framework is also based on a set of parameters that can be changed according to the user preference to suit the deployment scenario in consideration. The parameters are listed in Table II.

\section{B. Variables}

Our framework consists of several decision variables as detailed below.

- Binary variable $f_{i, j}$

$$
f_{i, j}= \begin{cases}1 ; & \text { if an OLT at the } i^{\text {th }} \text { CO is connected to } \\ & \text { a splitter at the } j^{\text {th }} \text { FAP } \\ 0 ; & \text { otherwise }\end{cases}
$$

- $\tilde{f}_{i, j}$ : number of fiber connections between the $i^{\text {th }}$ $\mathrm{CO}$ and the $j^{\text {th }} \mathrm{FAP}$

- Binary variable $d_{j, k}$

$$
d_{j, k}= \begin{cases}1 ; & \text { if a splitter at the } j^{t h} \mathrm{FAP} \text { is connected } \\ \text { to a ONU at the } k^{t h} \text { node } & \text { otherwise }\end{cases}
$$

- $\tilde{d}_{j, k}:$ number of fiber connections between the $j^{\text {th }}$ FAP and the $k^{t h}$ node

- Binary variable $s_{j}$

$$
s_{j}= \begin{cases}1 ; & \text { if } j^{t h} \text { FAP is selected to place splitter/s. } \\ 0 ; & \text { otherwise }\end{cases}
$$

- $\tilde{s}_{j}$ : number of splitters placed at the $j^{\text {th }}$ FAP.

- Binary variable $r_{k}$

$$
r_{k}= \begin{cases}1 ; & \text { if } k^{t h} \text { node is selected to place } \mathrm{RRH} / \mathrm{s} \\ 0 ; & \text { otherwise }\end{cases}
$$

- $\tilde{r}_{k}$ : number of RRHs placed at the $k^{t h}$ node.

- $x_{i}$ is a binary variable

$$
x_{i}= \begin{cases}1 ; & \text { if the } i^{\text {th }} \mathrm{CO} \text { is selected to place a BBU } \\ 0 ; & \text { otherwise }\end{cases}
$$

- $\tilde{y}_{i}$ is an integer variable: number of line cards in the $i^{\text {th }} \mathrm{CO}$.
- $h_{l}$ is a binary variable

$$
h_{l}= \begin{cases}1 ; & \text { if the } l^{\text {th }} \text { household is covered } \\ 0 ; & \text { otherwise }\end{cases}
$$

- $z_{k, l}$ is a binary variable

$$
z_{k, l}= \begin{cases}1 ; & \begin{array}{l}
\text { if } l^{t h} \text { household is served by an } \mathrm{RRH} \\
\text { placed at the } k^{\text {th }} \text { node }
\end{array} \\
0 ; & \text { otherwise }\end{cases}
$$

\section{The Total Deployment Cost of FWA Network}

The proposed framework considers the total deployment cost of the optical $\mathrm{x}$-haul and of the wireless access components of the FWA network. The optical $\mathrm{x}$-haul is designed by leveraging on the available fiber resources and by extending them to provide new fiber connectivity to RRHs when required. Therefore, this framework considers the cost associated with the use of the existing fiber facilities and also the deployment cost of new optical routes. The cost of using existing fiber facilities is calculated by considering the cost of the fiber itself, of the equipment, and of the labor. In particular, the feeder fiber routes are selected from a set of existing fiber routes. In the framework, the cost of using an existing fiber per unit length is denoted as $\alpha_{f f}$. The labor and equipment related costs involved in connecting a fiber $\mathrm{x}$-haul to an existing fiber access point is denoted by $\alpha_{f c}$. Finally, the cost of the optical time domain reflectometer (OTDR) that tests the integrity of existing fiber optic cables from the exchange/CO to the passive optical splitter/MUX is denoted as $\alpha_{f r}$.

Since new fiber network is required to be deployed from existing FAPs to some selected RRHs, the costs of new fiber, fiber preparation, their installations, and the cost of trenching need to be considered in the cost function. Therefore, in the framework, the cost to trench a unit length and the fiber installation cost per unit length are denoted by $\alpha_{f t}$ and $\alpha_{f i}$, respectively. The cost per unit length of a new fiber is denoted by $\alpha_{f b}$. The other major cost involved in x-haul deployment is arising from the deployment of equipment located in the CO. The transceiver cost is denoted by $\alpha_{o t}$. Each optical technology uses a different type of transceiver that can support the required bandwidth and therefore has a different value for $\alpha_{o t}$. The cost of a line card that supports $n_{p}$ number of PONs is denoted by $\alpha_{l c}$. Moreover, the cost of an OLT chassis is denoted by $\alpha_{o c}$. The framework also considers the cost associated with splitter/MUX installation for the scenario where the $\mathrm{x}$-haul consists of PtMP PON technology. The cost of a splitter/MUX and its installation are denoted by $\alpha_{s s}$ and $\alpha_{s i}$, respectively.

The costs associated with the deployment of a cell site are mainly related to the RRH cost, the price of an ONU, and the labor cost associated with the 
installation of this equipment. These costs are denoted by $\alpha_{r r}, \alpha_{o n}$, and $\alpha_{r i}$, respectively. $\alpha_{r r}$ can have a different value depending on the functional split used.

We also consider the cost associated with the BBU deployment at the selected central office(s). In the framework, the cost of a BBU that supports $n_{r b}$ number of RRHs is denoted by $\alpha_{b b}$. A BBU hotel that will be deployed at a selected $\mathrm{CO}$ consists of $n_{b b}$ number of BBUs. The labor cost associated with the installation of a BBU hotel is denoted by $\alpha_{b i}$.

\section{Objective}

The main objective of the framework is to minimize the total deployment cost of the 5G FWA network. The objective function of the framework is shown in Eq. (1) depicting each of the major cost categories under consideration.

$$
\begin{aligned}
& \underbrace{\min \alpha_{f r} \sum_{i \in \mathbf{C}} \sum_{j \in \mathbf{M}} \tilde{f}_{i, j}+\alpha_{f f} \sum_{i \in \mathbf{C}} \sum_{j \in \mathbf{M}} \tilde{f}_{i, j} * d f_{i, j}}_{\text {feeder fiber }}+ \\
& \underbrace{\left(\alpha_{f t}+\alpha_{f i}\right) \sum_{j \in \mathbf{M}} \sum_{k \in \mathbf{O}} d_{j, k} * d d_{j, k}+\alpha_{f b} \sum_{j \in \mathbf{M}} \sum_{k \in \mathbf{O}} \tilde{d}_{j, k} * d d_{j, k}}_{\text {distribution fiber }} \\
& +\underbrace{\alpha_{l c} \sum_{i \in \mathbf{C}} \tilde{y}_{i}}_{\text {ONU \& RRH }}+\underbrace{\left(\alpha_{b b}+\alpha_{b i}+\alpha_{o c}\right) \sum_{i \in \mathbf{C}} x_{i}}_{\text {BBU \& OLT chassis }}+ \\
& \underbrace{\left(\alpha_{\text {ot }}+\alpha_{f c}\right) \sum_{j \in \mathbf{M}} \tilde{s}_{j}+\underbrace{}_{s i} \sum_{j \in \mathbf{M}}+\alpha_{s s} \sum_{j \in \mathbf{M}} \tilde{s}_{j}}_{\text {ost per connection at CO cards }}+ \\
& \underbrace{\left(\alpha_{\text {on }}+\alpha_{r r}\right) \sum_{k \in \mathbf{O}} \tilde{r}_{k}+\alpha_{r i} \sum_{k \in \mathbf{O}} r_{k}}_{\text {splitters/MUX }}
\end{aligned}
$$

The objective function captures the cost arising from the existing feeder fiber usage, installing new distribution fiber, fiber connections at the central office for using existing fiber facility, in addition to the equipment and installation costs arising from RRHs and ONUs at the cell site, splitters/MUXs at the FAPs, and BBU, OLT and optical transceivers at the CO.

\section{E. Constraints}

Our framework consists of several constraints to guarantee the major requirements of both optical and wireless networks. These constraints capture the network requirements including household coverage, user capacity, limitations of split ratio of the splitters/MUX, PtP/PtMP optical connectivity, and BBU placement. The constraints of our framework are as follows.
1) Coverage Requirement:

$$
\begin{gathered}
\sum_{l \in \mathbf{H}} h_{l} \geq p_{c} * n_{B} / 100 \\
h_{l} \leq \sum_{k \in \mathbf{O}} t_{k, l} * r_{k}, \quad \forall l \in \mathbf{B} \\
h_{l} \geq \sum_{k \in \mathbf{O}} t_{k, l} * r_{k} / b i g M, \forall l \in \mathbf{B}
\end{gathered}
$$

One major requirement of a 5G FWA network is to provide enough network coverage for houses/buildings in the considered area. Therefore, we need to guarantee that the number of houses covered by $5 \mathrm{G}$ cells meets the FWA requirement. Here we consider the deployment of omnidirectional antenna at the cell site. The household coverage is modeled through cell radius with the use of the parameter $t_{k, l}$. The parameter $t_{k, l}$ has different values depending on the radius we considered for the deployment. The constraints of required coverage are formulated as shown in Eqs. (2), (3), and (4). These equations make sure that $p_{c} \%$ of houses are covered in the considered area. $p_{c}$ can be set according to the preference of service providers.

\section{2) Capacity Requirement:}

$$
\begin{gathered}
\tilde{r}_{k} * c_{r} \geq c_{h} * \sum_{l \in \mathbf{B}} z_{k, l}, \forall k \in \mathbf{O} \\
\sum_{k \in \mathbf{O}} z_{k, l} \geq h_{l}, \quad \forall l \in \mathbf{B} \\
z_{k, l} \leq t_{k, l}, \quad \forall k \in \mathbf{O}, \forall l \in \mathbf{B}
\end{gathered}
$$

Another major requirement of the FWA service is to provide sufficient wireless bandwidth to fixed users (ex. household) to provide guaranteed services. Therefore, the capacity requirement of the network should also be considered at the planning stage of the network. In order to ensure the capacity requirement of a fixed user $\left(c_{h}\right)$, our framework incorporates constraints shown in Eqs.(5), (6), and (7).

3) Point to Multipoint Deployment:

$$
\begin{gathered}
\sum_{i \in \mathbf{C}} \tilde{f}_{i, j}==\tilde{s}_{j}, \forall j \in \mathbf{M} \\
\sum_{j \in \mathbf{M}} \tilde{d}_{j, k}==\tilde{r}_{k}, \forall k \in \mathbf{O} \\
\sum_{j \in \mathbf{M}} d_{j, k}==r_{k}, \forall k \in \mathbf{O} \\
d_{j, k} \leq s_{j}, \forall j \in \mathbf{M}, \forall k \in \mathbf{O}
\end{gathered}
$$




$$
\sum_{k \in \mathbf{O}} \tilde{d}_{j, k} \leq n_{s} * \tilde{s}_{j}, \forall j \in \mathbf{M}
$$

Depending on the considered functional split, optical technology used in $\mathrm{x}$-haul varies to support the required transport bandwidth. For example, whilst functional split Options 8 and 7.1 require $P t P$ optical connectivity, other functional split options can be supported through high bandwidth PON technologies. In order to provide a single framework that can offer the cost-optimal solution irrespective of optical technology in use, we designed the connectivity constraints that are suitable for PtMP network. The cost optimal solution for PtP $\mathrm{x}$-ahul option can be obtained by changing a few parameters such as setting $n_{s}=1$ and modifying related cost values in the objective function.

In a PtMP PON network, splitters/MUXs are placed at the selected FAP location. Each of these splitters requires one connection to the OLT/CO. Similarly, each ONU placed at a selected location of a node needs an optical connectivity from a splitter. This point to multipoint connectivity requirement is ensured by the constraints shown in Eqs. (8 - 11). Moreover, the limitation of split ratio $\left(1: n_{s}\right)$ is considered for each deployment and the corresponding number of fiber connections are guaranteed by Eq. (12).

\section{4) Network Span:}

$$
\begin{aligned}
f_{i, j} * d f_{i, j}+d_{j, k} * d d_{j, k} & \leq d_{\max }, \\
& \forall i \in \mathbf{C}, \forall j \in \mathbf{M}, \forall k \in \mathbf{O}
\end{aligned}
$$

Passive optical networks typically span across tens of $\mathrm{kms}$. The span of the network is also dependent on the number of splits and the RAN requirement. Therefore, in our framework, the maximum span (the total length of distribution and feeder fibers) of the $\mathrm{x}$-haul is captured using Eq. (13).

5) Line Card Selection at the CO :

$$
\begin{gathered}
y_{i} \leq\left(\sum_{j \in \mathbf{M}} \tilde{f}_{i, j} / n_{p}\right)+1, \forall i \in \mathbf{C} \\
y_{i} \geq \sum_{j \in \mathbf{M}} \tilde{f}_{i, j} / n_{p}, \forall i \in \mathbf{C}
\end{gathered}
$$

Each OLT line card supports only $n_{p}$ number of PONs and this requirement is captured by Eqs. (14) and (15).

6) Placement of $B B U$ :

$$
\begin{gathered}
y_{i} / b i g M \leq x_{i}, \forall i \in \mathbf{C} \\
y_{i} \geq x_{i}, \forall i \in \mathbf{C}
\end{gathered}
$$

Since we consider the deployment of an entire FWA network, our framework places a BBU in the $i^{t h}$ $\mathrm{CO}$ location if the $i^{\text {th }} \mathrm{CO}$ location is selected for the deployment of OLTs. This constraint is enforced by Eqs. (16) and (17).

7) Nonlinear Relationships between Variables:

$$
\begin{gathered}
\tilde{f}_{i, j} \geq f_{i, j}, \forall i \in \mathbf{C} \forall j \in \mathbf{M} \\
\tilde{f}_{i, j} / b i g M \leq f_{i, j}, \forall i \in \mathbf{C} \forall j \in \mathbf{M} \\
\tilde{d}_{j, k} \geq d_{j, k}, \forall j \in \mathbf{M} \forall k \in \mathbf{O} \\
\tilde{d}_{j, k} / b i g M \leq d_{j, k}, \forall j \in \mathbf{M} \forall k \in \mathbf{O} \\
\tilde{s}_{j} \geq s_{j}, \forall j \in \mathbf{M} \\
\tilde{s}_{j} / b i g M \leq s_{j}, \forall j \in \mathbf{M} \\
\tilde{r}_{k} \geq r_{k}, \forall k \in \mathbf{O} \\
\tilde{r}_{k} / b i g M \leq r_{k}, \forall k \in \mathbf{O}
\end{gathered}
$$

There are integer and binary variable pairs which have nonlinear relationships. These pairs are $\left(\tilde{f}_{i, j}\right.$ and $\left.f_{i, j}\right),\left(\tilde{d}_{j, k}\right.$ and $\left.d_{j, k}\right),\left(\tilde{s}_{j}\right.$ and $\left.s_{j}\right)$, and $\left(\tilde{r}_{k}\right.$ and $\left.r_{k}\right)$. These non-linear relationships are captured by the linear constrains shown in Eqn. (18) to (25). For example, the binary variable $r_{k}$ represents the placement of $\mathrm{RRHs} / \mathrm{ONUs}$ at the $k^{\text {th }}$ node where $k \in \mathbf{O}$ and the integer variable $\tilde{r}_{k}$ represents the number of RRHs/ONUs placed at the $k^{\text {th }}$ node. These two variables have a non-linear relationship, $r_{k}=\min \left(1, \tilde{r}_{k}\right)$, which is captured by the pair of constraints shown in Eqs. (24) and (25).

8) Bounds of Integer Decision Variables:

$$
\begin{gathered}
\tilde{f}_{i, j} \geq 0, \forall i \in \mathbf{C}, \forall j \in \mathbf{M} \\
\tilde{d}_{i, j} \geq 0, \forall j \in \mathbf{M}, \forall k \in \mathbf{O} \\
0 \leq r_{k} \leq n_{r}, \forall k \in \mathbf{O} \\
\tilde{s}_{j} \geq 0, \forall j \in \mathbf{M}
\end{gathered}
$$

\section{Evaluations of The Optimal Solutions}

In order to demonstrate its applicability, the proposed framework is used to plan a 5G FWA with different optical x-haul options in a suburban area in eastern Australia. We choose the size of the geographical area accordingly with the standard planning area used by service providers. We use the commercially available CPLEX linear programming solver (version 12.6.2.0) to find the optimal deployment solution. It is important to note that depending on the complexity and the size of the dataset, the solution time varies. However, for the use case considered in this work CPLEX is able to solve the problem in a few seconds on a computer with 18GB RAM and Intel i7 processor.

The map of the considered use case is illustrated in Fig. 2(a). This suburban area has over 6000 residents. 


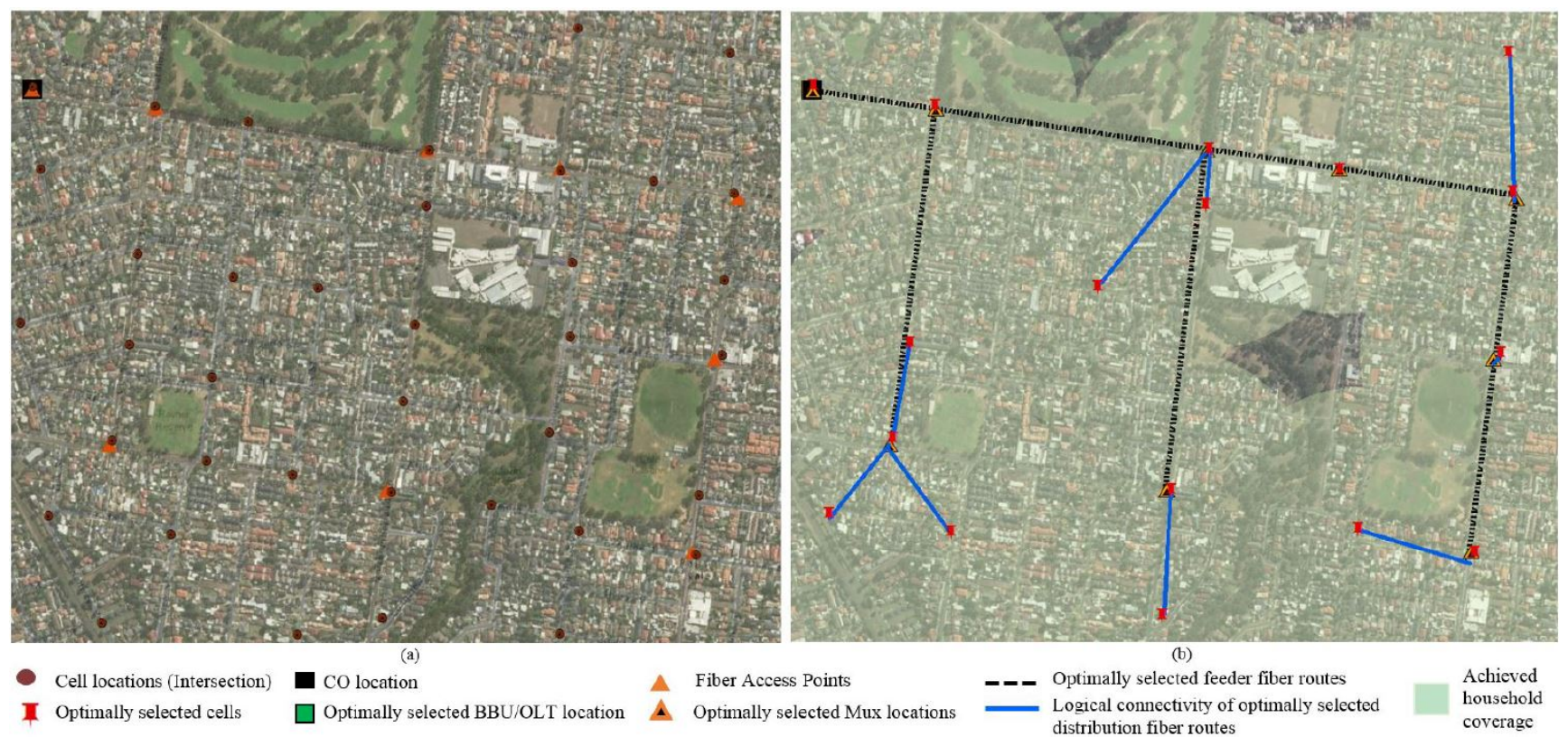

Fig. 2. (a) Input dataset; (b) Optimal solution of 10 DWDM-PON 1:8 split x-haul network and 99\% household coverage with 25Mbps capacity requirement.

The major intersections available in this suburban area are considered to be the most viable places for the placement of RRH/antennas, as they are typically equipped with a light pole. Existing COs of a key telecommunication service provider in Australia are considered as the potential locations of BBU and OLT placement and in the considered area we have only one CO location. Moreover, placement of feeder and distribution fibers are selected from the pre-defined networks of existing fibers and new routes, respectively. The existing fiber access points are considered as potential locations of splitters/MUXs placement and they are connected to the $\mathrm{CO}$ by existing fiber routes.

We consider various cost components in modeling the objective function. Their notation and cost are listed in Table III. The cost values are chosen from various studies and vendor specification materials

TABLE III Cost Components: 10G DWDM-PON BASED X-HAUL

\begin{tabular}{lcc}
\hline \hline Cost component & Notation & Normalized Cost \\
\hline Existing fiber $/ \mathrm{km}$ & $\alpha_{f f}$ & 41.6 \\
Distribution fiber/ km & $\alpha_{f d}$ & 10.4 \\
Fiber trenching/ km & $\alpha_{f t}$ & 93.7 \\
Testing per fiber & $\alpha_{f r}$ & 1 \\
OLT chassis (16 slots) & $\alpha_{o c}$ & 118.75 \\
OLT line card (16X10G) & $\alpha_{l c}$ & 13.3 \\
Optical transceiver & $\alpha_{o t}$ & 3.6 \\
CO connection & $\alpha_{f c}$ & 3.1 \\
MUX/DMUX (1:8) & $\alpha_{s i}$ & 3.3 \\
ONU & $\alpha_{o n}$ & 4.7 \\
RRH & $\alpha_{r r}$ & 20.8 \\
BBU & $\alpha_{b b}$ & 729.1 \\
\hline \hline
\end{tabular}

[22]-[25] and are normalized with respect to the cost of fiber testing. We compare the optimal deployment costs when different $\mathrm{x}$-haul options are used with diverse capacity and coverage requirements of households. Each optical $x$-haul option can support different functional split options. For example, a 10G DWDM PON with 1:4 splits can support functional split options 1 to 6 and 7.3 whilst a $40 \mathrm{G}$ TWDM PON with $1: 4$ splits can support options 1 to $6,7.2$, and 7.3.

The capacity that can be supported by an RRH is assumed to be $1 \mathrm{Gbps}$, whilst in order to provide realistic analyses, the capacity required per household is varied from $25 \mathrm{Mbps}$ to $100 \mathrm{Mbps}$ (i.e., the capacity ratio of RRH to household is varied from 1:40 to 1:10). However, the capacity and population coverage requirements are parameters in the framework and can be set according to the specification of the equipment and service providers. Moreover, $n_{r}$ and $n_{p}$ can also be set according to the desired specifications. In our study, we use 3 and 16, respectively.

Each deployment scenario under evaluation provides a unique optimal deployment solution derived from the framework. For example, Fig. 2(b) shows the map of the optimal solution when the $\mathrm{x}$-haul consists of a 10G DWDM PON, the RRHs have $300 \mathrm{~m}$ radius, the coverage requirement is set to $99 \%$, and the capacity required per household is set to $25 \mathrm{Mbps}$. The feeder fibers showed in Fig. 2(b) (i.e., dotted lines) are optimally selected from the set of existing fiber routes. The blue lines correspond to the logical connectivity of optimally selected distribution fiber routes that connect the optimally selected RRHs with the selected MUX locations. The information of which feeder fiber route and which cell location are to be used, in addition the 


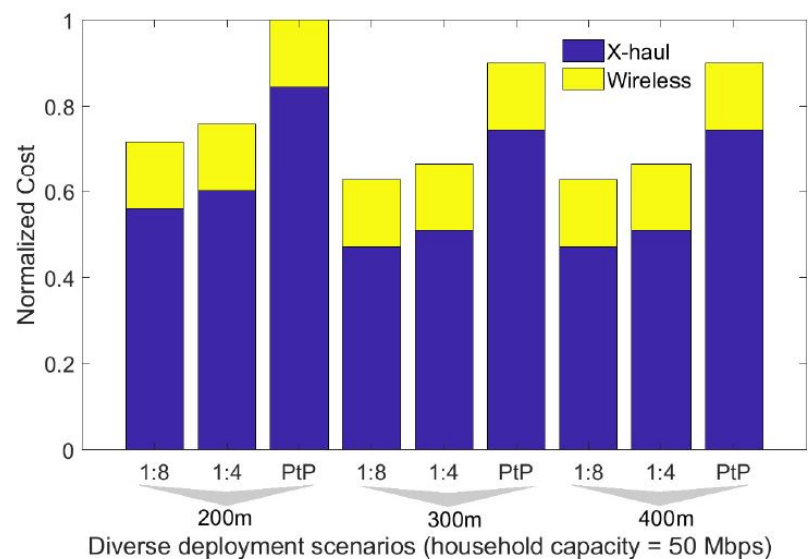

Fig. 3. Optimal deployment costs with diverse cell radii.

locations where a MUX and OLT/BBU should be placed are all derived by solving the problem formulation.

We compare the total deployment costs of different FWA deployment scenarios. Figure 3 shows the optimal deployment costs (i.e., broken down to the cost contributions of the wireless and the optical $\mathrm{x}$ haul segments) for three $\mathrm{x}$-haul options (i.e., PtP and 10G DWDM-PON with different split ratios) and for different values of the cell radii. The cost values shown in Fig. 3 are normalized with respect to the total deployment cost result for the case of a PtP fiber x-haul with a cell radius of $200 \mathrm{~m}(\approx 1790 \mathrm{k})$. The coverage and the capacity requirements of households are set to $99 \%$ and $50 \mathrm{Mbps}$, respectively.

According to the results shown in Fig. 3, the x-haul is the major cost contributor to the FWA deployment. When the cell radius reduces, the total deployment cost increases irrespective of the $\mathrm{x}$-haul technology under consideration. Particularly, when the cell radius is increased from $200 \mathrm{~m}$ to $300 \mathrm{~m}$ there is a significant cost reduction in the total deployment costs. On the other hand, moving from the $300 \mathrm{~m}$ to the $400 \mathrm{~m}$ deployment scenarios, the deployment cost remains

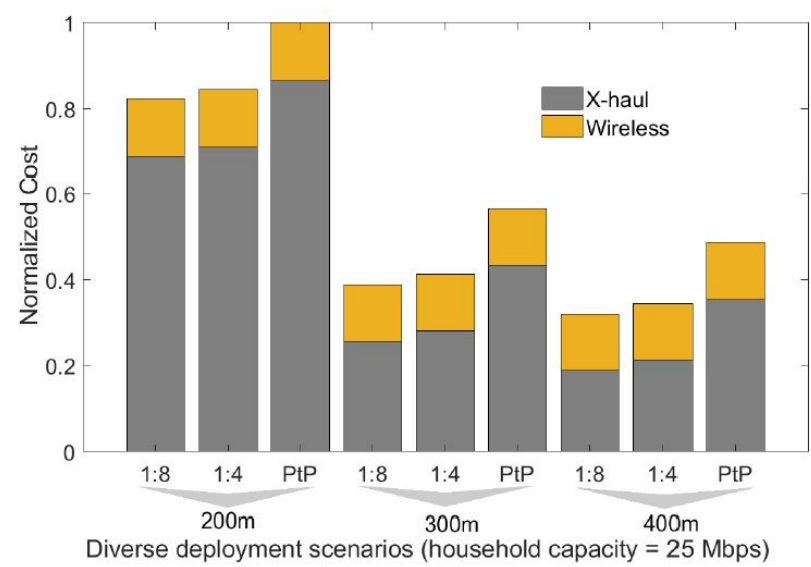

Fig. 4. Optimal deployment costs with diverse cell radii. the same. However, for all radii, PON-based solutions show significant cost reduction compared to the PtP case. For example, when the FWA network has a $200 \mathrm{~m}$ cell radius, 10G DWDM-PON with a 1:8 split ratio can save approximately $30 \%$ of the deployment cost compared to the PtP deployment scenario.

The results shown in Fig. 3 also exhibit dependence of the $\mathrm{x}$-haul deployment cost on the functional split options. For example, 10G DWDM PON with 1:8 splitting ratio provides an average bandwidth of $1.25 \mathrm{Gbps}$ per cell which matches the $\mathrm{x}$-haul bandwidth requirements of functional split Options 1 to 6. Therefore, for a $200 \mathrm{~m}$ cell radius deployment, 10G DWDM PON with 1:8 splitting ratio leads to the optimal cost for functional split Options 1 to 6. Further, for functional split Option 7.3, an x-haul bandwidth of 2 Gbps is required. Under this condition, a 10G DWDM PON with 1:4 splitting ratio results in the lowest cost. On the other hand, functional split Option 7.2 can be implemented using 10G PtP fiber links as Option 7.2 requires 6 Gbps of bandwidth. As shown in Fig. 3 under $200 \mathrm{~m}$ cell radii, using any functional split Option from 1 to 6 (i.e., 1:8 splitting ratio) and/or Option 7.3 (i.e., 1:4 splitting ratio) can save $34 \%$ and $29 \%$ of the x-haul deployment cost, respectively. These numbers are in comparison to the usage of functional split Option 7.2.

Figure 4 shows the normalized deployment costs for a scenario similar to the one considered in Fig. 3 but with a different household capacity requirement (25Mbps). The cost values shown in Fig. 4 are normalized with respect to the total deployment cost result for the case of a PtP fiber $\mathrm{x}$-haul with a cell radius of $200 \mathrm{~m}$ $(\approx 1330 \mathrm{k})$. The results in Fig 4 show trends similar to those in Fig. 3. However, the value of the cell radius has a different impact on the total deployment cost. In particular, in the case of 1:8 10G DWDM-PON x-haul, the total deployment cost can be halved using $300 \mathrm{~m}$ cell radii instead of $200 \mathrm{~m}$. These results clearly show the importance of developing a generalized optimization framework that provides cost-optimal deployment irrespective of the FWA network setting.

Next, we evaluate the total deployment costs when PtP fiber and 1:8 10G DWDM-PON are used as $\mathrm{x}$-haul with varying household coverage requirements. The capacity requirement is set to $50 \mathrm{Mbps}$. The results are shown in Fig. 5. The cost values are normalized with respect to the total deployment cost result for the case of a PtP fiber $\mathrm{x}$-haul with a coverage requirement of 99\%. The results shown in Fig. 5 indicate that the deployment costs of both $\mathrm{x}$-haul options are higher when the household coverage increases. However, with a $10 \mathrm{G}$ DWDM-PON the deployment cost is significantly lower compared to the PtP scenario and the cost difference is greater when the coverage requirement increases. For example, when the coverage requirement is set to $99 \%$, PON shows $30 \%$ reduction in the deployment cost compared to the PtP scenario. 


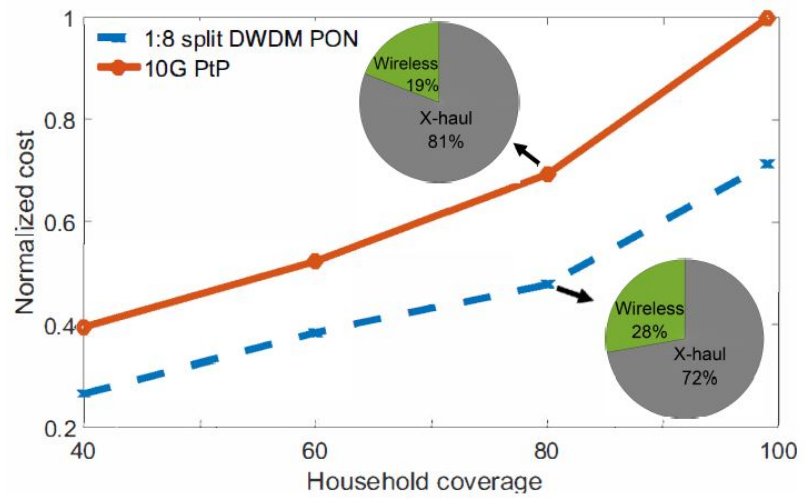

Fig. 5. Optimal deployment costs vs. coverage requirements.

To get a better understanding of the impact of the network parameters on the optimal solution, we compute the deployment cost when the household capacity requirement varies from $25 \mathrm{Mbps}$ to $100 \mathrm{Mbps}$. For this set of analyses, we assume the number of cells per location to be limited to 6 and the value of cell radius to be equal to $200 \mathrm{~m}$. The cost values shown in Fig. 6 are normalized with respect to the total deployment cost result for the case of a PtP fiber x-haul with a capacity requirement of $100 \mathrm{Mbps}(\approx 2450 \mathrm{k})$. The results shown in Fig. 6 indicate that the deployment cost of all the $\mathrm{x}$-haul options increases with the increasing value of the required household capacity. Also, there is only a slight difference between the deployment cost of the 1:4 and the 1:8 split ratio cases for the PONbased $\mathrm{x}$-haul options. On the other hand, there is a significant difference between the PON-based and PtP-based $x$-haul scenarios. More specifically, the cost difference is higher for higher values of the household capacity requirement. For example, when the capacity requirement is set to $100 \mathrm{Mbps}$ the PON-based $\mathrm{x}$-haul option can save more than $40 \%$ of the deployment cost compared to the PtP case.

We also evaluate and compare the value of the opti-

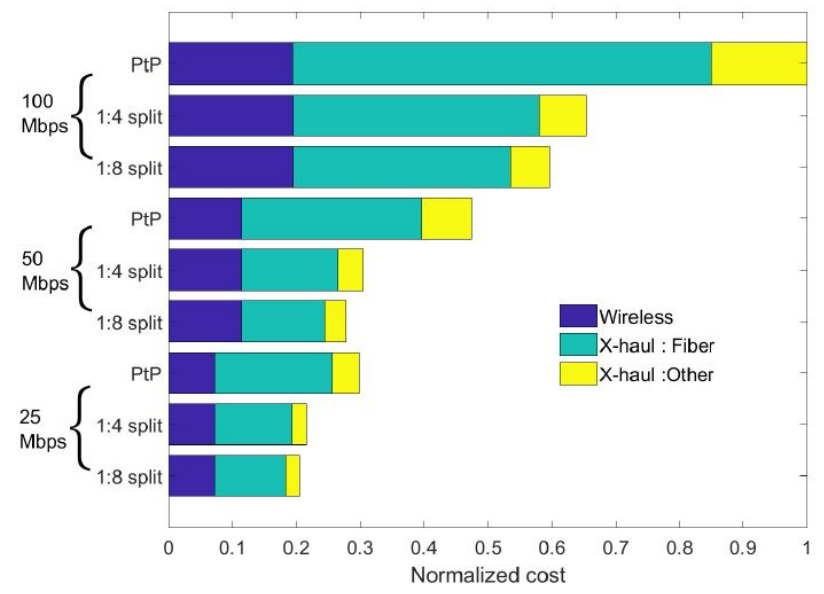

Fig. 6. Optimal deployment costs vs. capacity requirements. mal solution from the framework considering different types of PON technologies for the x-haul. Figure 7 shows the deployment costs when 40G TWDM PONs and 10G DWDM PONs are used for the $\mathrm{x}$-haul under different household capacity requirements and cell radii conditions. It is worthwhile to note that, when using TWDM PON which allows multiple users/cells to share the same wavelength similar to traditional TDM PON, extra care should be taken to minimize the operational delay. This delay is heavily dependent on the resource allocation mechanism implemented in the network. Previous studies have investigated the implementation of appropriate resource allocation mechanisms to meet the $5 \mathrm{G}$ delay requirements when using TDM-based PON [26] and also demonstrated the effectiveness of using TWDM PON as 5G x-haul [27].

The cost values shown in Fig. 7 are normalized with respect to the total deployment cost of 10G DWDM PON with $200 \mathrm{~m}$ cell radii and $50 \mathrm{Mbps}$ capacity requirement. Due to unavailability of the cost of $40 \mathrm{G}$ TWDM PON line card, we take a conservative approach where we assume only four 40G TWDM PONs can be supported by each line card instead of 16 in $10 \mathrm{G}$ DWDM PON. The results shown in Fig. 7 correspond to the deployment of 10G DWDM PON and 40G TWDM PON with 1:8 and 1:16 split ratio, respectively. As can be seen, $\mathrm{x}$-haul is the major contributor to the total deployment cost irrespective of the PON technology used and the deployment scenario considered. For low household rate requirement conditions, both PON technology options show similar values of the deployment cost, regardless of the considered cell radius. However, when the household capacity requirement is set to $50 \mathrm{Mbps}$, 40G TWDM PON shows slight improvement with the added advantage of providing higher capacity $x$-haul compared to 10G DWDM PON. This is due to the fact that when the capacity requirement is higher, the network needs more cells and $\mathrm{x}$ haul links. Therefore, a high capacity TWDM PON can cater to these requirement efficiently with its ability

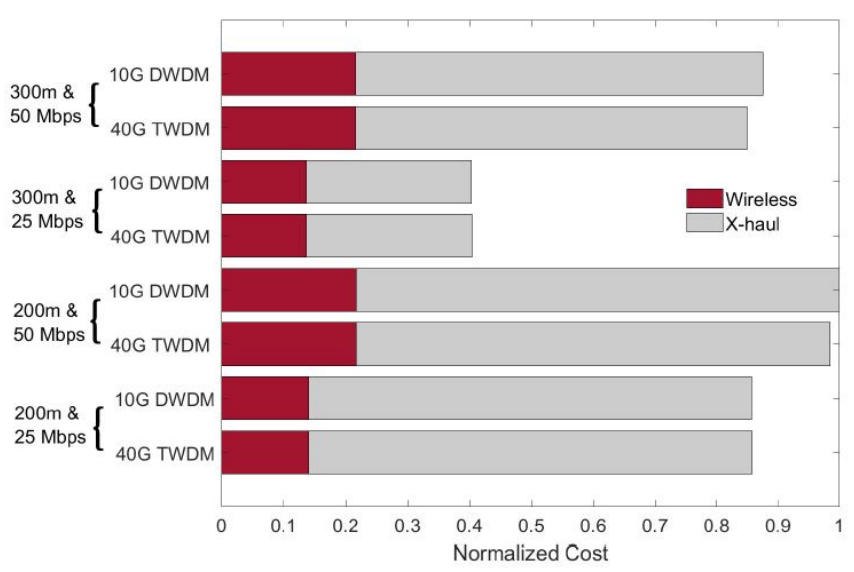

Fig. 7. Optimal deployment costs for different types of PONs. 
of providing a higher number of splits.

\section{CONCLUSION}

In this paper, we proposed a generalized optimization framework that can be used to cost-optimally plan 5G FWA and its optical x-haul network. Our proposed ILP-based framework is also capable of meeting important requirements of FWA network such as fixed user coverage and capacity. We demonstrated the suitability of our proposed framework by using it to plan $5 \mathrm{G}$ FWA and its optical x-haul networks in Australian eastern suburban areas. We analyzed the optimal deployment cost performance of the framework under various network conditions and deployment scenarios in order to demonstrate how versatile the proposed framework is in identifying, in each case, the best $\mathrm{x}$ haul option among the ones under consideration. The framework presented in this paper is one of the first to be able to jointly optimize the wireless and the optical $\mathrm{x}$-haul segment of a 5G FWA network and it will be vital to realizing future-proof fixed wireless broadband deployments.

\section{ACKNOWLEDGMENT}

This work is supported by ARC Discovery Project DP150101977, by the Group of Eight \& DAAD Australian-German Research Cooperation Grant Scheme, by the Early Carrier Research Grant funded by The University of Melbourne, and by the Kista $5 \mathrm{G}$ Transport Lab (K5) project funded by VINNOVA and Ericsson.

\section{REFERENCES}

[1] K. Laraqui, S. Tombaz, A. Furuskär, B. Skubic, A. Nazari, and E. Trojer, "Fixed wireless access on a massive scale with 5G," in Ericsson Technology Review, 2016.

[2] F. Boccardi, R. W. Heath, A. Lozano, T. L. Marzetta, and P. Popovski, "Five disruptive technology directions for 5G," in IEEE Communications Magazine, vol. 52, no. 2, pp. 74-80, 2014.

[3] Ericsson, "Fixed Wireless Access handbook, Extracted Version," in Handbook, 2018. Available: https://www.ericsson.com

[4] Next Generation Mobile Networks Alliance, "NGMN 5G White Paper," White Paper, 2015. Available: https://www.ngmn.org

[5] China Mobile Research Institute, C-RAN: The Road Towards Green RAN, White Paper, 2013.

[6] M. Peng, S. Yan, K. Zhang and C. Wang, "Fog-computing-based radio access networks: issues and challenges," in IEEE Network, vol. 30, no. 4, pp. 46-53, 2016.

[7] C. Ranaweera, E. Wong, C. Lim, A. Nirmalathas, and C. Jayasundara, "5G C-RAN with Optical Fronthaul: An Analysis from a Deployment Perspective," in IEEE / OSA Journal of Lightwave Technology, vol. 36, no.11, 2018.

[8] B. Skubic, M. Fiorani, S. Tombaz, A. Furuskär, J. Mårtensson, and P. Monti, "Optical transport solutions for 5G fixed wireless access," in IEEE/OSA Journal of Optical Communication and Networking, vol. 9, no. 9, pp. 10-18, 2017.

[9] F. Athley, S. Tombaz, E. Semaan, C. Tidestav, and A. Furuskär, "Providing Extreme Mobile Broadband using higher frequency bands, beamforming and Carrier Aggregation," in Proc. of IEEE PIMRC, 2015.

[10] X. Pang, M. Beltrán, J. Sánchez, E. Pellicer, J. J. V. Olmos, R. Llorente, and I. T. Monroy, "Centralized Optical-FrequencyComb-Based RF Carrier Generator for DWDM Fiber-Wireless Access Systems," in IEEE /OSA Journal of Optical communication and Networking, Vol.6, no.1, pp. 1-7, 2014
[11] C. U. Bas, R. Wang, T. Choi, S. Hur, K. Whang, J. Park, J. Zhang, and A. F. Molisch, "Outdoor to Indoor Penetration Loss at $28 \mathrm{GHz}$ for Fixed Wireless Access," in IEEE International Conference on Communications (ICC), Kansas City, MO, 2018.

[12] 3GPP, TR38.801, "Radio access architecture and interfaces (Release 14), 2016.

[13] D. Ha, D. Choi, H. Kim, J. Kum, J. Lee and Y. Lee, "Passive repeater for removal of blind spot in NLOS path for 5G fixed wireless access (FWA) system," in 2017 IEEE International Symposium on Antennas and Propagation \& USNC/URSI National Radio Science Meeting, San Diego, CA, 2017.

[14] M. Hashemi, M. Coldrey, M. Johansson, and S. Petersson, "Integrated Access and Backhaul in Fixed Wireless Access Systems," in IEEE 86th Vehicular Technology Conference (VTCFall), Toronto, 2017.

[15] S. Hurley, S. Allen, D. Ryan, and R. Taplin, "Modelling and planning fixed wireless networks," in Journal of Wireless Networks, vol. 16, no. 3, pp. 577-592, 2010.

[16] P. Ritthisoonthorn, K. Ahmed, and D. Krairit, "Planning of Efficient Wireless Access with IEEE 802.16 for Connecting Home Network to the Internet" in EURASIP Journal on Wireless Communications and Networking, 2010. DOI: https://doi.org/10.1155/2010/625414, 2010.

[17] B. Jaumard, S. Song, and R. Chowdhury, "Design and dimensioning of hybrid PONs," in Journal of Photonic Network Communications, vol. 31, no. 3, pp. 466-482, 2016.

[18] B. Jaumard, and R. Chowdhury, "Design and dimensioning of hybrid PONs," in Journal of Computer Communications, vol. 36, no. 14, pp. 1539-1551, 2013.

[19] J. Li and G. Shen, "Cost minimization planning for greenfield passive optical networks," Journal of Optical Communications and Networking, vol. 1, no. 1, pp. 17-29, 2009.

[20] A. Agata and K. Nishimura, "Suboptimal PON network designing algorithm for minimizing deployment cost of optical fiber cables," in $16^{\text {th }}$ International Conference on Optical Network Design and Modeling, pp. 1-6, 2012.

[21] C. Ranaweera, P. Monti, B. Skubic, M. Furdek, L. Wosinska A. Nirmalathas, C. Lim, and E. Wong, "Optical X-haul Options for 5G Fixed Wireless Access: Which One to Choose?," in IEEE Conference on Computer Communications (INFOCOM), Poster, HI, USA, April 2018.

[22] Fiber Transceiver Solution, Available: http://www.fiber-optictransceiver-module.com/cost-difference-between-mmf-and-smfoptics.html

[23] C. Ranaweera, M. G. C. Resende, K. C. Reichmann, P. P. Iannone, P. S. Henry, B-J. Kim, P. D. Magill, K. N. Oikonomou, R. K. Sinha, and S. L. Woodward, "Design and optimization of fiber optic small-cell backhaul based on an existing fiber-tothe-node residential access network," in IEEE Communications Magazine, vol.51, no.9, pp.62-69, 2013.

[24] COMBO (COnvergence of fixed and Mobile BrOadband access/aggregation networks) project, Available: http://www.ictcombo.eu/

[25] C. Ranaweera, C. Lim, A. Nirmalathas, C. Jayasundara, and E. Wong, "Cost-Optimal Placement and Backhauling of Small-Cell Networks," in IEEE / OSA Journal of Lightwave Technology, vol. 26, no. 2, pp. 3850-3857, 2015.

[26] N. Shibata, S. Kuwano, J. Terada, and H. Kimura, "Dynamic IQ data compression using wireless resource allocation for mobile fronthaul with TDM PON," in IEEE/OSA Journal of optical communication, vol. 7, no. 3, pp. A372A378, 2015.

[27] X. Wang et al., "Handover reduction in virtualized cloud radio access networks using TWDM-PON fronthaul," in IEEE/OSA Journal of Optical Communications and Networking, vol. 8, no. 12, pp. B124-B134, 2016. 